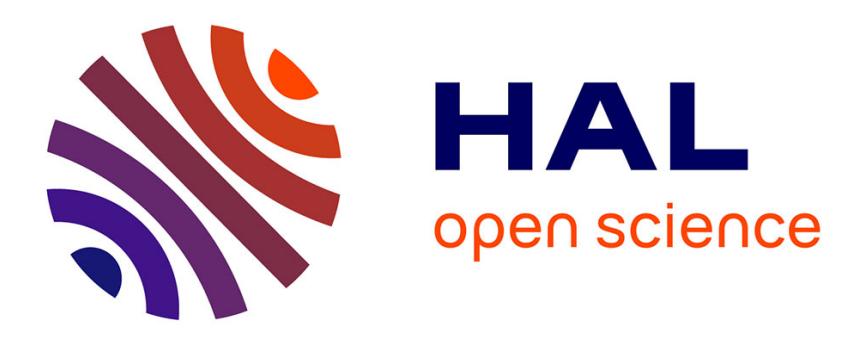

\title{
Pre-play communication in procurement auctions: silence is not golden \\ Lucie Ménager
}

\section{To cite this version:}

Lucie Ménager. Pre-play communication in procurement auctions: silence is not golden. 2015. hal00856078v2

\section{HAL Id: hal-00856078 \\ https://hal.science/hal-00856078v2}

Preprint submitted on 6 Mar 2015

HAL is a multi-disciplinary open access archive for the deposit and dissemination of scientific research documents, whether they are published or not. The documents may come from teaching and research institutions in France or abroad, or from public or private research centers.
L'archive ouverte pluridisciplinaire HAL, est destinée au dépôt et à la diffusion de documents scientifiques de niveau recherche, publiés ou non, émanant des établissements d'enseignement et de recherche français ou étrangers, des laboratoires publics ou privés. 


\title{
Pre-play communication in procurement auctions: silence is not golden.
}

\author{
Lucie Ménager*
}

This version: January, 2015

\begin{abstract}
I study the effect of cheap talk between bidders on the outcome of a first-price procurement auction in which participation is costly. Although no side-payments or commitments are allowed, their exists a family of equilibria in which sellers use communication to collude on a subset of participants and/or to reveal information about their cost. I show that the buyer may benefit from cheap talk between sellers, and that the surplus increases with the amount of information revealed in equilibrium under fairly general conditions. This is because when communication is cheap, sellers cannot directly collude on higher prices. Rather, communication leads to a competition between fewer, but more aggressive bidders, which entails more allocative efficiency and a decrease in the total wasteful entry cost.
\end{abstract}

JEL Classification: C72; D44; D82; L44.

Keywords: Cheap talk; Procurement auction; Collusion.

*LEMMA, Université Paris 2, 4 rue Blaise Desgoffe, 75006 Paris, lucie.menager@u-paris2.fr. This research was supported by the Banque de France Foundation Young Economist Prize and the French National Research Agency (ANR). I benefited from the useful comments of Olivier Bos, Nicolas Dromel, Alia Gizatulina, Jeanne Hagenbach, Frédéric Koessler, Rida Laraki, François Maniquet, Chantal Marlats, Tristan Tomala, Abhinay Muthoo, and seminar participants at the Paris School of Economics, Paris Game Theory Seminar (IHP), Bristol University, Université Lille 1, Cologne-Paris Theory Workshop, Workshop on Advances in Mechanism Design, and CORE. I am particularly indebted to Françoise Forges and Francesco Giovannoni, for enlightening comments, and to Achim Wambach who found a mistake in a previous version of the paper. The usual disclaimer applies. 


\section{Introduction}

Communication between bidders is one of the most important target of competition authorities. Most of them have developed guidelines ${ }^{1}$ to help governments improve public procurement by fighting bid rigging, according to which bidders have to vouch for they did not communicate with any competitor regarding prices, methods used to calculate prices, and the intention to submit a bid or not. The reason is the conventional wisdom in industrial organization, according to which communication between bidders in public procurement would 1) discourage competition, 2) increase public spending, and 3) decrease efficiency. In this paper, I show in a first-price procurement auction with entry that none of these points is true when communication is cheap talk. First, bidders cannot use cheap-talk messages to collude on higher prices. Communication only allows to coordinate on a subset of participants and/or to reveal information about individual costs. Second, communication need not lead to a higher price for the buyer. Finally, the surplus need not decrease when bidders are allowed to communicate, and even increases with the amount of information transmitted in equilibrium.

I consider a buyer who seeks to obtain an object by procuring it via a sealed-bid first-price reverse auction with entry. There are 3 potential sellers, who hold privately known costs of fulfilling the contract (say, of producing the object). Sellers have the option to pay a fixed and non-recoverable entry cost and bid a price, or to stay out of the competition. The entry cost can be interpreted either as a direct participation cost (travel expenses, participation fees,...), or as a bid preparation cost (time spent and resource allocated to preparing the bid, opportunity cost,...). Finally, if at least one seller participates in the auction, the contract is awarded to the seller submitting the lowest price, and payoffs are realized. Prior to bidding, bidders send one round of public messages to each other. The literature on collusion in auctions usually assumes the possibility of commitment and the existence of side payments. On the contrary, I assume cheap-talk communication: messages are costless, unverifiable and non binding. ${ }^{2}$

I show that, even with this simple communication structure, the game admits a family

\footnotetext{
${ }^{1}$ See for instance http://competitionbureau.gc.ca/eic/site/cb-bc.nsf/eng/00599.html

${ }^{2}$ Another way to model the effects of announcements is to suppose they are costly. A large literature (starting with Fudenberg and Tirole (1983), Sobel and Takahashi (1983)) analyzes how bargainers can improve their terms of trade by undertaking costly actions.
} 
of sequential equilibria in which realistic features of collusive behaviors emerge. In these equilibria, called $(\theta, \Phi)$-equilibria, bidders use communication for two reasons: 1$)$ to coordinate on a subset of participants, and/or 2) to reveal information about their cost. Collusion thus emerge in equilibrium as the combined effect of coordination and information sharing.

The construction of such equilibria works as follows. After the communication stage, a "cartel" of potential bidders is selected to participate in the auction on the basis of the message exchange. Members of the cartel participate if and only if their cost is below some maximal participating cost, which depends on the size of the cartel and on the information about each other's cost revealed by the message exchange. The contract is then awarded to the lowestcost bidder, among those who participate. In these equilibria, the way bidders are selected to participate only partially depends on their cost. Equilibrium strategies can then be interpreted in terms of "taking turns", and in this sense capture a relevant aspect of collusive agreements.

Because participation is endogenous, sellers have an obvious incentive to look more competitive than they actually are, which makes information revelation not straightforward in equilibrium. It occurs because the information a seller may credibly reveal through cheap talk has opposite effects on her expected payoff: "claiming to be weak", that is sending the same message as high-cost sellers, increases the probability one's opponents participate, but increases also the level of one's opponents' bids, and then one's probability of making a winning bid. "Claiming to be strong" has the opposite effects. Therefore, any information revelation about one's cost induces a trade-off between bidding positions and probability of participation. I show that in $(\theta, \Phi)$-equilibria, strong sellers credibly separate from "out-bidders", who never participate in the auction.

In $(\theta, \Phi)$-equilibria, the welfare implications of cheap-talk between bidders are not those expected by competition authorities. Communication decreases the number of potential bidders either directly, by selecting a cartel of participants, or indirectly, by decreasing the maximal participating cost. However, because of participation costs, fewer potential bidders need not decrease ex ante the revenue or the surplus. Perhaps more surprisingly, the surplus increases with the amount of information transmitted via communication under fairly general conditions. This comes from the fact that when it is cheap talk, communication between bidders does not decrease competition: rather, it leads to a competition between fewer, but more aggressive bidders, which results in a decrease in the total wasteful preparation cost and a 
better allocative efficiency.

The paper is organized as follows. The related literature is exposed in Section 2. Section 3 presents the procurement auction with pre-play communication. In section 4, I define, characterize, and prove the existence of $(\theta, \Phi)$-equilibria. I also show that communication strictly enlarges the set of equilibrium of the auction, in the sense that 1) there is no symmetric equilibrium of the auction in which bidders play the strategies played in a $(\theta, \Phi)$-equilibrium, and that 2) cheap-talk does more than replicating a public randomization device, because of information sharing. In section 5, I study the welfare properties of cheap-talk. Section 6 concludes and discusses the robustness of $(\theta, \Phi)$-equilibria to some assumptions of the model, and main proofs are gathered in the Appendix.

\section{Related literature}

There is few formal theory on the role of communication in collusion. Kandori and Matsushima (1998) and Compte (1998) explore the role of communication in repeated games with imperfect monitoring, in which the fact that players observe different sets of signals about other players' past actions makes collusion hardly sustainable. They assume the possibility for players to communicate at the end of each period, and prove a Folk theorem. Aoyagi (2007) studies collusion in repeated auctions when bidders report their private signals to a center, which then returns instructions to them based on the reported signal profile.

The closest work to ours is Campbell (1998), who studies non-committal coordination in second-price auction with entry, where coordination consists in playing sunspot or cheaptalk equilibria. He proves coordination can yield higher ex-post payoff to all bidders, and that coordination through cheap talk is more efficient than coordination through exogenously generated public information. Miralles (2010) generalizes Campbell's result to more-than-twobidders, more-than-one-object cases.

This paper is also related to the literature on communication in competitive bidding games. Matthews and Postlewaite (1987), and Farrell and Gibbons (1987) introduce cheap talk to bargaining games, in which a single buyer and a single seller bargain over an exchange price. Although such coordination is different from that between bidders competing on the same side of a market, they find the existence of equilibria in which players reveal information in the 
same way they do in $(\theta, \Phi)$-equilibria: low-value buyers and high-value sellers are willing to jeopardize continued negotiation so as to improve their bargaining position. The two parties use talk to trade-off bargaining positions against the probability of continued negotiation. More recently, Chakraborty and Harbaugh (2003) study a multi-issue bargaining game in which player $A$ sends a message to player $B$, who makes a "take-it-or-leave-it" offer to player $A$ after hearing the message. They show that while the two sides' interests are directly opposed on each issue, cheap talk can be credible if there is bundling over the two issues. In a different communication setting, Rieck (2010) studies signalling in a first-price auction with two bidders, where one of the two bidders has the option to release a signal about her cost when she learns it. He shows that a bidder may benefit from the presence of an informative signal about her own cost, if this signal is not too precise. Gonçalves (2008) studies the existence of a communication equilibrium in a model of a common-value English auction with discrete bidding.

\section{A procurement auction with pre-play communication}

\subsection{The procurement auction}

Consider a standard independent private values procurement auction with entry à la Samuelson (1985), shortly called auction in the rest of the paper. A single buyer seeks to obtain an indivisible object from 3 possible sellers via a sealed-bid first-price reverse auction. Let $\mathcal{N}=\{1,2,3\}$ denote the set of possible sellers. Under the procurement rules, the buyer accepts the lowest bid, provided it is below her value for the object $\rho>0$. Ties are resolved via uniform randomization, and losers of the auction obtain nothing. Sellers are risk-neutral and incur a privately known cost $t \in[0,1]$ of supplying the object. Costs are generated independently from the uniform ${ }^{3}$ distribution on $[0,1]$. Sellers have the option to pay a participation cost $k \leq 0$ and become active bidders, or to opt out and remain inactive. The status chosen by a seller is denoted by $s \in \mathcal{S}=\{$ Active, Inactive . The name bidder will refer to a seller

\footnotetext{
${ }^{3}$ In a previous version of the paper, I considered the general case of $N \geq 2$ sellers and a general distribution of costs on $[0,1]$. Since cheap talk introduces asymmetries between bidders at the bidding stage, strong assumptions had to be made on out-of-equilibrium beliefs to guarantee tractability. This more specified model allows a characterization of equilibria with light requirements on out-of-equilibrium beliefs, without limiting the coordination power of communication.
} 
who actually participates in the auction. After observing which of their opponents are active, ${ }^{4}$ bidders submit a bid $b \in \mathbb{R}_{+}$. Finally, inactive sellers have payoff 0 , and the buyer gets payoff 0 if no seller participates.

The auction has a unique symmetric equilibrium in strictly increasing bidding strategies, described in Samuelson (1985), called here the no-talk equilibrium. The participation strategy is a cost cutoff for which sellers are indifferent between participating and opting out. Above this cutoff, thus called the maximal participating cost, they do not participate and get 0 . This equilibrium cutoff is determined as follows. Consider a seller with $\operatorname{cost} \tau$ whose all opponents participate whenever their cost is below $\tau$. If she participates, she will have the highest cost among actual bidders. She will thus lose the auction and get $-k$ except in case she faces no bidder, which occurs if all opponents are above $\tau$. In this case, she bids the maximal price and gets $\rho-\tau$. Her expected payoff if she participates is thus $(\rho-\tau)(1-\tau)^{2}-k$. In a symmetric equilibrium, she must be indifferent between participating and opting out, which gives the equilibrium entry condition

$$
(\rho-\tau)(1-\tau)^{2}-k=0
$$

Let $\tau^{n t}$ denote the unique solution in $[0, \rho-k]$ to equation (1). Below $\tau^{n t}$, sellers participate and bid the optimal price in a first-price procurement auction in which bidders' costs are distributed on $\left[0, \tau^{n t}\right]$. Precisely, a cost- $t$ bidder facing $q$ opponents bids $b\left(t ; \tau^{n t}, q\right)$, with

$$
b(t ; \tau, q):= \begin{cases}t+\frac{\tau-t}{q+1} & \text { if } q \geq 1 \\ \rho & \text { if } q=0\end{cases}
$$

Her expected payoff is then $u\left(t ; \tau^{n t}, q\right)$ with

$$
u(t ; \tau, q):= \begin{cases}\left(\frac{\tau-t}{q+1}\right)\left(1-\frac{t}{\tau}\right)^{q}-k & \text { if } q \geq 1 \\ \rho-t-k & \text { if } q=0\end{cases}
$$

At the beginning of stage 2 , the number of actual bidders is a random variable. In the rest of the paper, I denote by $v(t ; \tau, n, \lambda)$ the expectation of $u(t ; \tau, q)$ conditional on $q$ following a binomial distribution $B(n-1, \lambda)$. In the no-talk equilibrium, the stage-2 expected payoff to seller $t$ is $v\left(t, \tau^{n t}, 3, \tau^{n t}\right)$.

\footnotetext{
${ }^{4}$ The implications of assuming that sellers might simultaneously decide about participation and bid are discussed in the Conclusion.
} 


\subsection{Cheap-talk pre-play communication}

Before the auction occurs a communication stage in which sellers talk to each other by sending messages chosen in a countable set $\mathcal{M}=\left\{m_{1}, m_{2}, \ldots\right\}$. The set of probability distributions on $\mathcal{M}$ is denoted $\Delta(\mathcal{M})$. I consider a simple communication structure: communication is cheap talk (costless, non binding and non verifiable), direct, simultaneous, public, and not repeated. In other words, sellers simultaneously send a unique, costless message to all other sellers.

The auction with pre-play communication is thus a three-stage game whose timing is as follows: a) in stage 1, sellers learn their cost and send a public message to each other, b) in stage 2 , they decide whether to enter on the basis of their cost and the message exchange, and c) in stage 3 , actual bidders bid according to their cost, the message exchange and the status of all sellers.

\section{Strategies}

A seller's strategy has three components: a message strategy $m:[0,1] \rightarrow \Delta(\mathcal{M})$, which determines what message to send as a function of her cost; a participation strategy $s:[0,1] \times$ $\mathcal{M}^{3} \rightarrow$ Active, Inactive $\}$, which prescribes whether to enter as a function of her cost and the message exchange; and a bid strategy $b:[0,1] \times \mathcal{M}^{3} \times\{$ Active, Inactive $\} \rightarrow \mathbb{R}_{+}$, which determines what to bid according to her cost, the message exchange and the participation decisions, after any history in which the seller is active in stage 3. The strategy of seller $i$ is denoted $\sigma_{i}=\left(m_{i}, s_{i}, b_{i}\right)$.

\section{Histories and beliefs}

Let $H$ denote the set of histories of the game, with $H^{k} \subseteq H$ the subset of histories of length $k$. Because players' actions are public, a seller's information set after history $h \in H^{k}$ consists of all histories $h^{\prime} \in H^{k}$ where her cost and publicly observable past actions are the same as in $h$. Sellers have a common prior belief on each other's cost, and hold a common belief on equilibrium paths. I denote by $\mu_{i}(h \mid \sigma)$ the belief held by seller $i$ on her opponents after history $h$ conditional on the strategy profile $\sigma:=\left(\sigma_{i}\right)_{i}$, and by $\mu(h \mid \sigma):=\left(\mu_{i}(h \mid \sigma)\right)_{i \in \mathcal{N}}$ the belief profile after $h$. A belief system $\mu(. \mid \sigma): H \rightarrow \Delta([0,1])^{N-1 \times N}$ specifies a belief profile conditional on $\sigma$ after any history in $H$. Finally, for any $h \in H, P(h \mid \sigma)$ denotes the probability of history $h$ conditional on $\sigma$. 


\section{Equilibrium concept}

I investigate the existence of symmetric sequential equilibria of the game, consisting of pairs $(\sigma, \mu)$ such that $(1) \sigma_{i}=\sigma_{j}$ for all $i, j,(2) \sigma$ is sequentially rational given $\mu$, and (3) $\mu$ is consistent with $\sigma$. Focusing on symmetric equilibria makes this analysis more restrictive than in Campbell (1998), where pre-play communication is shown to enforce coordination on particular asymmetric equilibria of a second-price auction. The reason of this restriction is the first-price setting, which makes the analysis of asymmetric equilibria barely tractable.

\section{$4 \quad(\theta, \Phi)$-equilibria}

As in any game with strategic communication, the game admits babbling symmetric equilibria, in which players consider cheap talk as meaningless and play the no-talk equilibrium strategies. More interestingly, the game also admits a family of non-babbling equilibria, in which players use communication for two reasons: 1) to transmit information about their competitiveness, and 2) to coordinate on some cartel of participants. Such equilibria are characterized by two parameters in a way that will be made precise in the section: a parameter $\theta$, part of players' message strategy, which represents the amount of information revealed by players through communication, and a parameter $\Phi$, part of players' status strategy, which represents the way players coordinate on a subset of participants. These equilibria are called $(\theta, \Phi)$-equilibria.

In this section, I define, characterize, and prove the existence of $(\theta, \Phi)$-equilibria. I emphasize the role of communication in equilibrium by showing that the mapping from costs to outcomes in a $(\theta, \Phi)$-equilibrium could not occur without communication.

\subsection{Definition}

Let us call any non-empty collection of players a cartel, and denote by $\mathcal{C}$ the set of possible cartels. The typical strategy played in a $(\theta, \Phi)$-equilibrium, called a $(\theta, M, \Phi)$-strategy, is characterized by a cutoff $\theta \in[0,1]$, a subset of messages $M \subseteq \mathcal{M}$, and a function $\Phi: \mathcal{M}^{N} \rightarrow \mathcal{C}$ which associates a cartel of potential bidders, namely sellers with a positive participation probability, to each message profile. According to this strategy, a seller below $\theta$ sends any message in $M$ with equal probability, and sends a message in $\mathcal{M} \backslash M$ otherwise. Put differently, 
sellers below $\theta$ credibly separate from sellers above $\theta$, but sellers below $\theta$ do not separate from each other. To the message profile $\mathbf{m}$ is associated a cartel $\Phi(\mathbf{m})$. Sellers outside $\Phi(\mathbf{m})$ do not enter, and potential bidders in $\Phi(\mathbf{m})$ participate if their cost is below the maximal participating cost, which depends on $|\Phi(\mathbf{m})|$, the number of potential bidders, and on $\theta$, the information revealed by the message exchange. In the last stage of the game, bidders play optimally given their beliefs and the subset of actual bidders. Because the probability to participate in the auction only partially depends on sellers' cost, $(\theta, M, \Phi)$-strategies can be interpreted in terms of "taking turns", and capture in this sense a relevant aspect of collusive agreements.

The construction of a $(\theta, \Phi)$-equilibrium works as follows. Sellers all play the same $(\theta, M, \Phi)$-strategy. When no seller deviates from $(\theta, M, \Phi)$, sellers update their beliefs according to Bayes rule. In the off-path event a seller outside the cartel of potential bidders participates, bidders believe that all costs, including the deviator's, are below the equilibrium maximal participating cost. This belief system, denoted $\mu^{*}$, is strongly consistent: concerning potential bidders, it is derived from Bayesian updating, since their entry is not a deviation. Concerning the deviator, $\mu^{*}$ puts a probability zero on an interval of costs for which it is dominated to enter together with the cartel.

Since the message support does not affect status and bid outcomes when sellers play $(\theta, M, \Phi)$, a $(\theta, \Phi)$-equilibrium refers to any sequential equilibrium in which sellers all play the same $(\theta, M, \Phi)$-strategy and update their beliefs according to $\mu^{*}$. Formally,

Definition $1\left((\theta, \Phi)\right.$-equilibrium). Let $\theta \in[0,1], \Phi: \mathcal{M}^{N} \rightarrow \mathcal{C}$, and $M$ a finite subset of $\mathcal{M}$. A $(\theta, \Phi)$-equilibrium is a sequential equilibrium where each seller $i$ plays and updates her beliefs as follows:

- At stage $1, i$ randomizes over $M$ if $t_{i} \leq \theta$, and plays in $\Delta(\mathcal{M} \backslash M)$ otherwise.

- At stage $2, i$ enters if and only if $i \in \Phi(\mathbf{m})$ and $t_{i} \leq \tau_{\theta,|\Phi(\mathbf{m})|}$, with $\tau_{\theta,|\Phi(\mathbf{m})|}$ determined by the equilibrium entry condition

$$
(\rho-\tau))\left(1-\frac{\tau}{\theta}\right)^{|\Phi(\mathbf{m})|-1}-k=0
$$

- At stage $3, i$ believes that all bidders' cost are smaller than $\tau_{\theta,|\Phi(\mathbf{m})|}$, and bids $b\left(t_{i} ; \tau_{\theta,|\Phi(\mathbf{m})|}, q\right)$ whenever she enters together with $q \geq 0$ other bidders. 


\subsection{Examples}

\section{Example 1}

This example shows that communication affects equilibrium outcomes even when sellers do not coordinate on entry coalitions. Fix some value $\theta \geq \rho-k$ and two messages $\underline{m}, \bar{m} \in \mathcal{M}$. At the communication stage, sellers above $\theta$ claim to be out by sending $\bar{m}$, and sellers below $\theta$ claim they might enter by sending $\underline{m}$. Other messages are sent with probability 0 . All sellers who sent $\underline{m}$ are then selected as potential bidders. After the message exchange, sellers below $\tau_{\theta, n}$ participate, with $n$ the number of potential bidders. Then they observe the identity of participants and assume that all bidders, including those who sent $\bar{m}$, are below $\tau_{\theta, n}$. Finally, a cost- $t$ bidder bids $b\left(t ; \tau_{\theta, n}, q\right)$ when she has $q$ opponents.

This $(\theta, \Phi)$-equilibrium is defined by the particular $(\theta, M, \Phi)$-strategy where $M=\{\underline{m}\}$ and $\Phi=\Phi_{\{\mathcal{N}\}}$ where $\Phi_{\{\mathcal{N}\}}(\mathbf{m}):=\left\{i \mid \mathbf{m}_{i}=\underline{m}\right\}$. This is the first-price analogous of the I'm-Out equilibrium in Campbell (1998), whose existence is proved in a second-price reverse auction. In this equilibrium, sellers above and below $\theta$ separate from each other by sending $\bar{m}$ for the former, and $\underline{m}$ for the latter. Then all are implicitly selected as potential bidders, and the contract is awarded to the lowest-cost bidder, among those who actually participate. The difference between an I'm-Out- and the $\left(\theta, \Phi_{\{\mathcal{N}\}}\right)$ - equilibrium is that, in the second-price setting, communication will only affect whether sellers participate, whereas the value of $\theta$ will also affect bid outcomes in a $\left(\theta, \Phi_{\{\mathcal{N}\}}\right)$-equilibrium.

\section{Example 2}

I now give an example of $(\theta, \Phi)$-equilibrium where seller 1 never competes against sellers 2 and 3. Fix some $\theta \geq \rho-k$. At the communication stage, sellers below $\theta$ claim they might enter by sending each of the messages $m_{1}$ and $m_{2}$ with probability $1 / 2$, and other messages with probability 0 . Sellers above $\theta$ claim to be out by sending a message in $\mathcal{M} \backslash\left\{m_{1}, m_{2}\right\}$. Then, they coordinate on a subset of potential bidders through the selection function $\widetilde{\Phi}$ defined as follows.

- If all say out, then all are selected as potential bidders (but will not participate in equilibrium).

- If all say out but seller $i$, then seller $i$ is the only potential bidder. 
- If only seller 1 says out, then sellers 2 and 3 are both potential bidders: $\widetilde{\Phi}\left(m, m^{\prime}, m^{\prime \prime}\right)=\{2,3\}$ for all $m \notin\left\{m_{1}, m_{2}\right\}, m^{\prime}, m^{\prime \prime} \in\left\{m_{1}, m_{2}\right\}$.

- If only seller 2 says out, then sellers 1 and 3 coordinate on the monopoly of $\{1\}$ when they send the same message, and on the monopoly of $\{3\}$ otherwise: $\widetilde{\Phi}\left(m_{1}, m, m_{1}\right)=\widetilde{\Phi}\left(m_{2}, m, m_{2}\right)=\{1\}$ and $\widetilde{\Phi}\left(m_{1}, m, m_{2}\right)=\widetilde{\Phi}\left(m_{2}, m, m_{1}\right)=\{3\}$ for all $m \notin\left\{m_{1}, m_{2}\right\}$.

- If only seller 3 says out, then sellers 1 and 2 coordinate on the monopoly of $\{1\}$ when they send the same message, and on the monopoly of $\{2\}$ otherwise:

$\widetilde{\Phi}\left(m_{1}, m_{1}, m\right)=\widetilde{\Phi}\left(m_{2}, m_{2}, m\right)=\{1\}$ and $\widetilde{\Phi}\left(m_{1}, m_{2}, m\right)=\widetilde{\Phi}\left(m_{2}, m_{1}, m\right)=\{2\}$ for all $m \notin\left\{m_{1}, m_{2}\right\}$.

- When no seller says out, then the message sent by seller 3 is not relevant: sellers coordinate on the monopoly of $\{1\}$ when sellers 1 and 2 send the same message, and on the duopoly $\{2,3\}$ otherwise:

$\widetilde{\Phi}\left(m_{1}, m_{1}, m\right)=\widetilde{\Phi}\left(m_{2}, m_{2}, m\right)=\{1\}$ and $\widetilde{\Phi}\left(m_{1}, m_{2}, m\right)=\widetilde{\Phi}\left(m_{2}, m_{1}, m\right)=\{2,3\}$ for $m \in\left\{m_{1}, m_{2}\right\}$.

After the message exchange, a potential bidder participates whenever she is below the maximal participating $\operatorname{cost} \tau_{\theta,|\widetilde{\Phi}(\mathbf{m})|}$. Then sellers observe the identity of participants and assume that all bidders, including those not selected by $\widetilde{\Phi}$, are below $\tau_{\theta,|\widetilde{\Phi}(\mathbf{m})|}$. Finally, a cost- $t$ bidder bids $b\left(t ; \tau_{\theta,|\widetilde{\Phi}(\mathbf{m})|}, q\right)$ when she has $q$ opponents.

Let us explain why these two strategies are sequential equilibria strategies. First, sellers excluded from the set of potential bidders (hereafter potential deviators) have no incentive to participate. Indeed, consider a potential deviator with $\operatorname{cost} 0$ (seller $\left.i_{D}\right)$, who would thus win with probability 1 . Suppose there are $n \in\{1,2\}$ potential bidders and that $i_{D}$ enters. If she enters alone, she bids (and gets) $\rho$. If $q \in\{1, n\}$ among these $n$ potential bidders also enter, she bids (and get with probability 1 ) $\frac{\tau_{\theta, n}}{q+1}$. Her expected payoff of participating is

$$
V_{i_{D}}(n):=v\left(0, \tau_{\theta, n}, n+1, \frac{\tau_{\theta, n}}{\theta}\right)
$$

In the next section, I show this expression is negative for all $n \in\{1,2\}$, provided that the entry cost is high enough, and that enough information is transmitted by communication. 
Under these conditions, any potential deviator prefers to opt out.

Second, given that only potential bidders participate, sellers have no profitable deviation from this message strategy. Sellers above $\rho-k$ are out-bidders, who stay out even when all their opponents do so. Out-bidders (among which sellers above $\theta$ ) are thus indifferent between all message strategies. Sellers below $\rho-k$, however, strictly prefer being selected as potential bidders. Therefore, they send a message in $M$ with probability 1 (with $M=\{\underline{m}\}$ in example 1 and $M=\left\{m_{1}, m_{2}\right\}$ in example 2$)$.

Finally, in example 2, messages $m_{1}$ and $m_{2}$ entail the same distribution function on $\mathcal{C}$ for both sellers. Let us first consider seller 1 . According to $\widetilde{\Phi}$, seller 1 is a potential bidder only when $\widetilde{\Phi}$ selects the cartel $\{1\}$. If seller 1 sends $m_{1}$, this happens when seller 2 sends $m_{1}$, or if seller 2 says out and seller 3 sends $m_{1}$, or when both sellers say out. If seller 1 sends $m_{2}$, this happens when seller 2 sends $m_{2}$ or when seller 2 says out and seller 3 sends $m_{2}$. Conditional on seller 2 and 3 saying out when they are above $\theta$, and sending $m_{1}$ and $m_{2}$ with probability $1 / 2$ otherwise, the probability that $\{1\}$ is selected is thus $1-\theta+\frac{\theta^{2}}{2}$ whether seller 1 sends $m_{1}$ or $m_{2}$. Let us now consider seller 2 . According to $\widetilde{\Phi}$, seller 2 is a potential bidder when $\widetilde{\Phi}$ selects $\{2\}$ or $\{2,3\}$. Yet $\widetilde{\Phi}$ selects $\{2\}$ with probability $(1-\theta)^{2}+\frac{\theta}{2}(1-\theta)$ and $\{2,3\}$ with probability $(1-\theta) \theta+\frac{\theta^{2}}{2}$ regardless of seller 2's message. The same reasoning shows that seller 3 is indifferent between $m_{1}$ and $m_{2}$.

\subsection{Characterization}

In this section, I give conditions on $\Phi, M, \theta$, and $k$ which characterize a $(\theta, \Phi)$-equilibrium. The first condition is necessary for the message strategy prescribed by $(\theta, M, \Phi)$ to be a bestresponse to $(\theta, M, \Phi)$. It enforces separation of low-cost sellers from out-bidders by imposing that messages out of $M$ preclude selection by $\Phi$. Sellers below $\rho-k$ are not indifferent among all cartels in $\mathcal{C}$. For randomization over $M$ to be a best-response for them, $\Phi$ must have the property that all messages in $M$ induce the same probability distribution on $\mathcal{C}$. With such selection functions, sellers cannot improve their chances of being selected in a cartel through their choice of message in $M$. Denoting $p_{i}^{(\theta, M, \Phi)}(c \mid m)$ the probability that $\Phi$ selects the cartel $c$ when $i$ sends $m$, conditional on $i$ 's opponents playing $(\theta, M, \Phi)$, condition $\mathbf{C} 1$ is stated as follows.

Condition C1 For all $i$, 
1) $i \in \Phi(\mathbf{m}) \Rightarrow \mathbf{m}_{i} \in M$

2) for all $c \in \mathcal{C}, p_{i}^{(\theta, M, \Phi)}(c \mid m)=p_{i}^{(\theta, M, \Phi)}\left(c \mid m^{\prime}\right)$ for all $m, m^{\prime} \in M$.

The last two conditions are necessary to preclude deviations from the participation strategy prescribed by $(\theta, M, \Phi)$. Condition $\mathbf{C 2}$ bounds the amount of information that can be transmitted by communication in equilibrium. First, $\theta$ must be higher than $\rho-k$, namely the cost above which opting out is a dominant strategy. If not, sellers in $[\theta, \rho-k]$, who strictly prefer participating alone rather than opting out, would deviate from $(\theta, M, \Phi)$ after histories in which all sellers say out. Second, $\theta$ must be smaller than some cutoff $\bar{\theta}$. Indeed, conditional on a set of potential bidders, an increase in $\theta$ has two positive effects on the expected payoff to a potential deviator: it increases the level of equilibrium bids, and thus what the deviator can get in case of competition, and decreases the participation probability of potential bidders, which also increases the deviator's expected payoff. Thus, $\theta$ must be small enough to give potential deviators a negative expected payoff and no incentive to enter:

Condition C2 $\theta \in[\rho-k, \bar{\theta}]$, with $\bar{\theta}$ solution of

$$
2 \theta^{2}-\frac{3}{2} \theta \rho+\frac{\rho^{2}}{2}-2 \theta k+\left(\theta-\frac{\rho}{2}\right) \sqrt{(\theta-\rho)^{2}+4 \theta k}=0
$$

C2 implies that $\theta$ measures the informativeness of communication: the lowest $\theta \in[\rho-k, \bar{\theta}]$, the more out-bidders are identified by communication, and thus removed from the set of potential bidders by their opponents.

Finally, if the entry cost is too small, no out-of-equilibrium beliefs could ever deter a potential deviator with cost zero to enter. Mathematically, condition C3 guarantees that $\rho-k \leq \bar{\theta}$ :

Condition C3 $k \geq \underline{k}$, with $\underline{k}>0$ solution ${ }^{5}$ of

$$
\rho^{2}+4 k^{2}-\frac{9}{2} k \rho+\frac{\rho-2 k}{2} \sqrt{-3 k^{2}+4 k \rho}=0
$$

A positive entry cost is clearly one of the motives underlying collusion, since it is the reason why losers of the auction strictly regret their entry decision. C3 shows that a relatively high entry cost is also necessary for collusion to occur.

\footnotetext{
${ }^{5} \underline{k}$ belongs to $] \frac{1}{3} \rho, \frac{2}{5} \rho[$.
} 
Conditions $\mathbf{C 1}, \mathbf{C 2}$, and $\mathbf{C 3}$, together characterize $(\theta, \Phi)$-equilibria.

Proposition 1 (Characterization). Consider the strategy profile $\sigma=((\theta, M, \Phi))_{i}$ and the belief system $\mu^{*} .\left(\sigma, \mu^{*}\right)$ is a symmetric sequential equilibrium of the game if and only if $\boldsymbol{C} \mathbf{1}$, C2, and C3 hold.

\subsection{Existence}

Existence of $(\theta, \Phi)$-equilibria has been proven in section 4.2. Here I show that, more generally, for any subset of cartels $C \subseteq \mathcal{C}$, a message subset $M_{C}$ and a selection function $\Phi_{C}$ can be constructed such that $\Phi_{C}$ and $M_{C}$ satisfy C1. Under conditions C2 and C3, $\left(\theta, M_{C}, \Phi_{C}\right)$ is a $(\theta, \Phi)$-equilibrium strategy.

Proposition 2. For every non-empty subset of cartels $C \in \mathcal{C}$, there exists a finite message set $M_{C}$ such that a corresponding $(\theta, \Phi)$-equilibrium obtains.

Let us show how such pairs $\left(\Phi_{C}, M_{C}\right)$ can be constructed. Fix an arbitrary subset of cartels $C \subseteq \mathcal{C}$ containing $\nu_{C} \geq 1$ elements. The message support of the strategy consists of the first $\nu_{C}$ messages in $M$, namely $M_{C}:=\left\{m_{1}, \ldots, m_{\nu_{C}}\right\}$. For $\mathbf{C} 1$ to be satisfied, $\Phi_{C}$ must select only sellers who send a message in $M_{C}$. Formally, for any message profile $\mathbf{m}, \Phi_{C}(\mathbf{m})$ must belong to the refinement of $C$ by $\mathbf{m}$, denoted $C \wedge \mathbf{m}$, obtained by excluding out-bidders from $C$ as follows:

$$
C \wedge \mathbf{m}:=\bigcup_{c \in C} c \cap\left\{i \mid \mathbf{m}_{i} \in M_{C}\right\}
$$

When $C \wedge \mathbf{m}$ is empty, then $\Phi_{C}(\mathbf{m})=\mathcal{N}$ : sellers do not coordinate through $\Phi_{C}$. When $C \wedge \mathbf{m}$ is a singleton, then $\Phi_{C}(\mathbf{m})$ consists of this singleton. When $C \wedge \mathbf{m}$ contains more than 2 elements, it must be the case that at least two sellers sent a message in $M_{C}$. Denote by $i_{1}$ and $i_{2}$ the first two of these sellers. ${ }^{6}$ The value of $\Phi_{C}(\mathbf{m})$ uniquely depends on $i_{1}$ and $i_{2}$ 's messages as follows:

$$
\Phi_{C}(\mathbf{m}):=a\left(\mathbf{m}_{i_{1}}, \mathbf{m}_{i_{2}}\right) \text { where }
$$

\footnotetext{
${ }^{6}$ Formally, $i_{1}:=\min \left\{i \mid \mathbf{m}_{i} \in M_{C}\right\}$ and $i_{2}:=\min \left\{i \neq i_{1} \quad \mid \mathbf{m}_{i} \in M_{C}\right\}$.
} 
- $a\left(m_{1}, m_{q}\right) \in C \wedge \mathbf{m}$ for all $q \in\left\{1, \nu_{C}\right\}$;

- $a\left(m_{p}, m_{q}\right)=a\left(m_{p-1}, m_{q+1}\right)$ if $p \in\left\{2, \nu_{C}\right\}$, and $q \in\left\{1, \nu_{C}-1\right\}$;

- $a\left(m_{p}, m_{\nu_{C}}\right)=a\left(m_{p-1}, m_{1}\right)$ if $p \in\left\{2, \nu_{C}\right\}$.

By construction, $\Phi_{C}$ and $M_{C}$ satisfy C1. To see this, it is convenient to represent the function $a$ by a $\nu_{C} \times \nu_{C}$-matrix $A$ whose typical element $A_{p q}$ is the cartel selected by $\Phi_{C}$ when sellers $i_{1}$ and $i_{2}$ respectively send $m_{p}$ and $m_{q}$. With $a_{q}$ standing for $a\left(m_{1}, m_{q}\right):^{7}$

$$
A:=\left(\begin{array}{cccc}
a_{1} & a_{2} & \ldots & a_{\nu_{C}} \\
a_{2} & a_{3} & \ldots & a_{1} \\
\vdots & \vdots & \vdots & \vdots \\
a_{\nu_{C}} & a_{1} & \ldots & a_{\nu_{C}-1}
\end{array}\right)
$$

Consider seller $i_{1}$. If her opponents randomly choose a message in $M_{C}$ whenever their cost is below $\theta$, all message profiles have the same probability conditional on $i_{1}$ 's message. Since lines of $A$ are permutations of the first line, any cartel appears the same number of times in all lines of $A$. Therefore, the probability distribution on $\mathcal{C}$ does not depend on $i_{1}$ 's message. Focus now on seller $i_{2}$. By construction, permutations are such that each cartel appears the same number of time in all columns of $A$, so that the same argument applies for $i_{2}$. Finally, other sellers have no influence on the probability distribution on $C$.

Note that this construction allows various probability distributions on $\mathcal{C}$. Consider for instance the case when $\nu_{C}=3$ and $C \wedge \mathbf{m}=\left\{c_{1}, c_{2}\right\}$. The matrices $A$ and $A^{\prime}$ where

$$
A:=\left(\begin{array}{ccc}
c_{1} & c_{2} & c_{1} \\
c_{2} & c_{1} & c_{1} \\
c_{1} & c_{1} & c_{2}
\end{array}\right) \text { and } A^{\prime}:=\left(\begin{array}{ccc}
c_{1} & c_{2} & c_{2} \\
c_{2} & c_{2} & c_{1} \\
c_{2} & c_{1} & c_{2}
\end{array}\right)
$$

clearly represent different selection functions, inducing different ex-ante probabilities on $\mathcal{C}$.

\footnotetext{
${ }^{7}$ Note that I impose no restriction on the $a_{q}$ 's, and that some of them, if not all, can be equal.
} 


\subsection{Cheap talk matters}

I now emphasize that cheap talk not only enlarges the set of Nash equilibria of the auction, but also the set of public correlated equilibria.

In any $(\theta, \Phi)$-equilibrium with $\Phi \neq \Phi_{\{\mathcal{N}\}}$, the mapping from costs to entry decisions is random. In particular, if a seller is not selected in a cartel, her entry decision will not depend on her cost, which cannot occur in a Nash equilibrium of the game without talk. This may, however, occur in a public correlated equilibrium. As a matter of fact, a strategy-proof selection function works exactly as a public randomization device: since players mix over a set of possible messages, the selection function can be interpreted as a random variable whose realization prescribes whether to enter or not. However, because $\theta<1$ in equilibrium, players also reveal information through communication, and the mapping from costs to outcomes (entry and bid decisions) could not occur in a correlated equilibrium with a public randomization device.

Proposition 3 (Cheap talk matters). The equilibrium mapping from costs to outcomes in $a(\theta, \Phi)$-equilibrium differs from that in the no-talk equilibrium.

As a proof, I compare the payoffs in the $(\theta, \widetilde{\Phi})$ and in the no-talk equilibrium. In the no talk-equilibrium, sellers participate whenever their cost is below $\tau^{n t}$. Then they get the expected payoff of sellers facing 3 potential bidders, that is

$$
V^{n t}(t)= \begin{cases}v\left(t, \tau^{n t}, 3, \tau^{n t}\right) & \text { if } t \leq \tau^{n t} \\ 0 & \text { if } t>\tau^{n t}\end{cases}
$$

In the $(\theta, \widetilde{\Phi})$-equilibrium, seller 1 is the only bidder whenever she participates. If her cost is above $\rho-k$, she does not enter and gets 0 . If her cost is below $\rho-k$, she enters whenever she is selected as potential bidder, which happens with probability $1-\theta+\frac{\theta^{2}}{2}$, and then gets the monopolist payoff $\rho-t-k$. Therefore, $(\theta, \widetilde{\Phi})$ yields seller 1 an interim payoff (before the cheap-talk phase) of

$$
V_{1}^{(\theta, \widetilde{\Phi})}(t)= \begin{cases}(\rho-t-k)\left[1-\theta+\frac{\theta^{2}}{2}\right] & \text { if } t \leq \rho-k \\ 0 & \text { if } t>\rho-k\end{cases}
$$


Since sellers 2 and 3 may compete against each other, their expected payoff depends on $\tau_{\theta, 2}$, the maximal participating cost among two potential bidders. If their cost is above $\rho-k$, they do not enter and get 0 . If their cost is in $\left[\tau_{\theta, 2}, \rho-k\right]$, they participate only when they are the unique potential bidder, which happens with probability $(1-\theta)\left(1-\frac{\theta}{2}\right)$, and then get the monopolist payoff. If their cost is below $\tau_{\theta, 2}$, they enter also when $\{2,3\}$ is selected, which occurs with probability $\theta\left(1-\frac{\theta}{2}\right)$, and then get the duopolist expected payoff $v\left(t, \tau_{\theta, 2}, 2, \frac{\tau_{\theta, 2}}{\theta}\right)$. Their interim payoff is therefore

$$
V_{2}^{(\theta, \widetilde{\Phi})}(t)=V_{3}^{(\theta, \widetilde{\Phi})}(t)= \begin{cases}(\rho-t-k)(1-\theta)\left(1-\frac{\theta}{2}\right)+v\left(t, \tau_{\theta, 2}, 2, \frac{\tau_{\theta, 2}}{\theta}\right) \theta\left(1-\frac{\theta}{2}\right) & \text { if } t \leq \tau_{\theta, 2} \\ (\rho-t-k)(1-\theta)\left(1-\frac{\theta}{2}\right) & \text { if } t \in\left[\tau_{\theta, 2}, \rho-k\right] \\ 0 & \text { if } t>\rho-k\end{cases}
$$

Finally, the ex-ante probability of participation depends on $\theta$ in a $(\theta, \Phi)$-equilibrium. For instance, probabilities of participation in the $(\theta, \widetilde{\Phi})$-equilibrium are $(\rho-k)\left(1-\theta+\frac{\theta^{2}}{2}\right)$ for seller 1 , and $\left(1-\frac{\theta}{2}\right)\left(\tau_{\theta, 2}+(1-\theta)\left(\rho-k-\tau_{\theta, 2}\right)\right)$ for sellers 2 and 3 . This emphasizes that, because of information sharing, cheap talk does more than replicating a public randomization device.

\section{Welfare issues}

In a $(\theta, \Phi)$-equilibrium, communication decreases the number of potential bidders either directly, by selecting a subset of participants, or indirectly, by decreasing the maximal participating cost. In a standard private values auction, the effect of a decrease in the number of potential participants is straightforward: both the revenue and the surplus will decrease in expectation. Any form of collusion would then decrease the revenue (to zero if there is only one bidder), and the surplus (through the loss of allocative efficiency). These classical results do not hold ${ }^{8}$ when bidders face participation costs. Indeed, a decrease in the number of potential bidders has then two effects on the revenue: a positive one, through the increase in the probability that at least two bidders participate, and a negative one, through the increase in equilibrium bids. It also has opposite effects on the surplus. On the negative side, it increases

\footnotetext{
${ }^{8}$ See Menezes and Monteiro (2000) for the trade-off in the expected revenue, and Samuelson (1985) for the result on the surplus.
} 
the value of the smallest cost, which hurts the allocative efficiency of the auction. On the positive side it decreases the total amount of participation costs paid by bidders.

The result of these trade-offs depends on the values of all parameters, and no general result can be given. Nonetheless, I provide examples showing that communication may decrease, but also, increase, the revenue and the surplus. Furthermore, I show that the surplus increases with the amount of information transmitted by communication for most selection functions, and for any selection function provided $\rho$ is not too high. This emphasizes that competition is not entirely characterized by the number of competitors. Indeed, the transmission of more information leads to a competition between fewer, but more aggressive bidders, which entails a decrease in the total wasteful participation cost and a better allocative efficiency.

\subsection{Communication and revenue}

The buyer's ex-post revenue is her valuation minus the winning bid, whenever trade occurs. If no seller participates, the revenue is 0 . If one seller participates, she bids the maximal price and the revenue is also 0 . If $q \in\{2,3\}$ sellers participate, the expected winning bid is the expected value of the second smallest cost among $q$ bidders, given that their cost is smaller than the maximal participating $\operatorname{cost} \tau$. The (expected) revenue conditional on $q \in\{2,3\}$ bidders and a maximal participating cost $\tau$ is thus $R(q, \tau):=\int_{0}^{\tau}(\rho-t) q(q-1) \frac{t}{\tau^{2}}\left(1-\frac{t}{\tau}\right)^{q-2} d t$, that is $R(2, \tau)=\rho-\frac{2}{3} \tau$ and $R(3, \tau)=\rho-\frac{\tau}{2}$.

Without communication, the maximal participating cost is always $\tau^{n t}$. The revenue is thus the expectation of $R\left(q, \tau^{n t}\right)$

$$
R^{n t}=3\left(\tau^{n t}\right)^{2}\left(1-\tau^{n t}\right)\left(\rho-\frac{2}{3} \tau^{n t}\right)+\left(\tau^{n t}\right)^{3}\left(\rho-\frac{\tau^{n t}}{2}\right)
$$

In a $(\theta, \Phi)$-equilibrium, the number of potential bidders and, consequently, the maximal participating cost, are random. The revenue is thus the expectation of $R\left(q, \tau_{\theta, n}\right)$, where $q$ and $n$ are both random variables.

The fact that communication may hurt the revenue if sellers coordinate through a very selective function is quite intuitive. In the $(\theta, \widetilde{\Phi})$-equilibrium for instance, the revenue is 0 unless sellers 2 and 3 are potential bidders, which happens with probability $\theta^{2}\left(1-\frac{\theta}{2}\right)$. The revenue is thus $R^{(\theta, \widetilde{\Phi})}=\theta^{2}\left(1-\frac{\theta}{2}\right)\left(\frac{\tau_{\theta, 2}}{\theta}\right)^{2} R\left(2, \tau_{\theta, 2}\right)$, that is

$$
R^{(\theta, \widetilde{\Phi})}=\left(1-\frac{\theta}{2}\right) \tau_{\theta, 2}^{2}\left(\rho-\frac{2}{3} \tau_{\theta, 2}\right)
$$


Taking $^{9}$ for instance $\rho=\frac{5}{4}, k=\frac{1}{2}$ and $\theta=\frac{3}{4}$, ex-ante revenues are $R^{(\theta, \widetilde{\Phi})} \simeq 0.074$ and $R^{n t} \simeq 0.2$.

Less intuitively, the buyer may also benefit from communication. Consider for instance the $\left(\theta, \Phi_{\{\mathcal{N}\}}\right)$-equilibrium, in which all sellers below $\theta$ are potential bidders. Here, the revenue is the expectation of $R\left(q, \tau_{\theta, 3}\right)$ if all sellers are potential bidders, which occurs with probability $\theta^{3}$, and the expectation of $R\left(q, \tau_{\theta, 2}\right)$ if only two of them are potential bidders, which occurs with probability $3 \theta^{2}(1-\theta)$. Therefore,

$$
R^{\left(\theta, \Phi_{\mathcal{N}}\right)}=3(1-\theta) \tau_{\theta, 2}^{2}\left(\rho-\frac{2}{3} \tau_{\theta, 2}\right)+3 \tau_{\theta, 3}^{2}\left(\theta-\tau_{\theta, 3}\right)\left(\rho-\frac{2}{3} \tau_{\theta, 3}\right)+\tau_{\theta, 3}^{3}\left(\rho-\frac{\tau_{\theta, 3}}{2}\right)
$$

With the same set of parameters, calculation gives $R^{(\theta, \widetilde{\Phi})} \simeq 0.6$.

\subsection{Communication and surplus}

Consider $n$ potential bidders, and let $t_{(1)}^{\theta, n}$, the smallest of the $n$ seller costs on $[0, \theta]$. Since transfers between both sides of the market can be ignored, the ex-post surplus is 0 if no seller participates, which occurs if $t_{(1)}^{\theta, n}>\tau_{\theta, n}$, and is $\rho-t_{(1)}^{\theta, n}-k \sum_{i} \mathbb{1}_{t_{i} \leq \tau_{\theta, n}}$ if at least one seller participates, which occurs when $t_{(1)}^{\theta, n} \leq \tau_{\theta, n}$. Thus the stage 2 surplus

$$
w(\theta, n):=\int_{0}^{\tau_{\theta, n}}(\rho-t) \frac{n}{\theta}\left(1-\frac{t}{\theta}\right)^{n-1} d t-n \frac{\tau_{\theta, n}}{\theta} k
$$

Without communication, the number of potential bidder is 3 and $\theta=1$, so that the (ex-ante) surplus in the no-talk equilibrium is

$$
W^{n t}=w(1,3)=\int_{0}^{\tau^{n t}}(\rho-t) 3(1-t)^{2} d t-3 \tau^{n t} k
$$

In a $(\theta, \Phi)$-equilibrium, the surplus is the expectation of $w(\theta, n)$

$$
W^{(\theta, \Phi)}=\sum_{n=1}^{3} P^{(\theta, \Phi)}(n) w(\theta, n)
$$

where $P^{(\theta, \Phi)}(n)$ is the probability that $n$ potential bidders are selected in equilibrium. The distribution function $P^{(\theta, \Phi)}$ is entirely characterized by the collection $\left\{f_{i}^{n}, i=1,2,3, n \leq i\right\}$, where $f_{i}^{n}$ stands for the probability that $\Phi$ selects $n$ potential bidders when $i \geq n$ sellers claim not to be out. ${ }^{10}$ For instance, $P^{\left(\theta, \Phi_{\{\mathcal{N}\}}\right)}$ is characterized by $f_{1}^{1}=f_{2}^{2}=f_{3}^{3}=1$. With this

\footnotetext{
${ }^{9}$ These parameters satisfy $\mathbf{C 2}$ and $\mathbf{C 3}$ and imply the following values for maximal participating costs: $\tau^{n t} \simeq 0.28, \tau_{\theta, 2} \simeq 0.34$ and $\tau_{\theta, 3} \simeq 0.23$.

${ }^{10}$ Note that the $\left(f_{i}^{n}\right)$ 's do not depend on $\theta$.
} 
notation,

$$
\begin{aligned}
& P^{(\theta, \Phi)}(1)=1-P(i=0)-f_{2}^{2} P(i=2)-\left(f_{3}^{2}+f_{3}^{3}\right) P(i=3) \\
& P^{(\theta, \Phi)}(2)=f_{2}^{2} P(i=2)+f_{3}^{2} P(i=3) \\
& P^{(\theta, \Phi)}(3)=f_{3}^{3} P(i=3)
\end{aligned}
$$

I first emphasize that a more aggressive coordination may not hurt the surplus. Indeed, for $\rho=\frac{5}{4}, k=\frac{1}{2}$ and $\theta=\frac{3}{4}, w(\theta, 1) \simeq 0.375, w(\theta, 2) \simeq 0.314, w(\theta, 3) \simeq 0.307$ and $W^{n t} \simeq 0.287$. It follows that $W^{(\theta, \Phi)}>W^{n t}$ for all $\Phi$. This is in line with Samuelson (1985)'s examples in which the surplus may decrease with the number of potential participants.

The effect of more information transmission on the surplus is not straightforward. First, the expected number of potential bidders is not monotonic in $\theta$. Second, even for a given number of potential bidders, the impact of an increase in $\theta$ on $w(\theta, n)$ is ambiguous. On the positive side, it decreases ${ }^{11}$ the individual participation probability $\frac{\tau_{\theta, n}}{\theta}$, and hence the total participation cost. On the negative side, it increases the value of the smallest valuation, which hurts the allocative efficiency of the auction.

For "monotonic" selection functions, according to which $f_{i}^{n}$ decreases with $i$, the overall effect of a decrease in $\theta$ is positive. In particular, at the "I'm Out" equilibrium in which all inbidders are potential bidders, the surplus increases with the amount of information transmitted by sellers. For other (and somehow less natural) selection functions, the result holds provided an upper bound on the buyer's valuation. Intuitively, if $\rho$ is relatively so large that sellers are nearly identical, increasing the number of bidders conveys little benefit in terms of allocative efficiency, but brings along the polar risks of inducing too many bidders (and then too much wasteful preparation cost) or no bidder at all, if the maximal participating cost decreases so much that no seller participates.

Proposition 4 (Efficiency-enhancing effect of information). Consider some selection function $\Phi$ and $f_{1}^{1}, f_{2}^{1}, f_{2}^{2}, f_{3}^{1}, f_{3}^{2}, f_{3}^{3}$ the associated conditional probabilities.

- If $f_{3}^{1} \leq f_{2}^{1}$, then $W^{(\theta, \Phi)}$ decreases with $\theta$ on $[\rho-k, \bar{\theta}]$.

- If $f_{3}^{1}>f_{2}^{1}$, then $\rho<3$ guarantees that $W^{(\theta, \Phi)}$ decreases with $\theta$ on $[\rho-k, \bar{\theta}]$.

\footnotetext{
${ }^{11}$ Indeed, $\frac{\partial}{\partial \theta} \frac{\tau_{\theta, n}}{\theta}=-\frac{\tau_{\theta, n}\left(\theta-\tau_{\theta, n}\right.}{\theta^{2}\left(\theta-\tau_{\theta, n}+\left(\rho-\tau_{\theta, n}\right)(n-1)\right)}$.
} 


\section{Concluding remarks}

This paper studies the impact of cheap-talk communication between sellers in a procurement auction with entry. I show the existence of a family of equilibria in which sellers use communication to coordinate on a subset of participants and to reveal information about their cost. I show that the buyer may benefit from cheap talk between sellers, and that the surplus increases with the amount of information transmitted in equilibrium under fairly general conditions. I do not think theses results advocate authorizing communication between sellers in public procurements, though. Clearly, if communication is not cheap, sellers can either collude on higher prices, which indeed increases public spending, or on bid rotation schemes which are generally inefficient. Rather, I think these results emphasize how different can be the outcome implications of cheap-talk and binding communication.

The existence of $(\theta, \Phi)$-equilibria crucially depends on a large enough entry cost. This gives a flavor of costly signalling to our cheap-talk setting. Indeed, sellers learn information about others from the observation of their entry decision, which indeed costs $k>0$ : entry is the real and costly signal about sellers' cost. It also crucially depends on the assumption that bidders observe the status of their opponents before bidding. Imagine that sellers simultaneously decide whether to enter and what to bid, and consider a seller, say $i$, whose cost is below but close to $\rho-k$. There is a profitable deviation from $(\theta, M, \Phi)$ for $i$, which prescribes to send a message in $\mathcal{M} \backslash M$, and stay out unless the selection function selects only one potential bidder, say $j$. Thinking she has no opponent, $j$ will enter and bid $\rho$. Knowing that, $i$ enters and wins the contract by bidding $\rho-\varepsilon$. Consequently, if sellers do not observe each other's status before bidding, the only symmetric equilibrium of the game is the no-talk equilibrium. This gives a theoretical foundation to the competition authorities' recommendation according to which sellers should not be allowed to communicate about their intention to participate.

Finally, sellers communicate in this model with one unique round of public and simultaneous messages. Even though this simple communication setting is enough for realistic features of collusive behaviors to appear in equilibrium, it is legitimate to wonder whether adding a mediator would enlarge the set of equilibria. The presence of a mediator in this particular collusion setting could be deemed not realistic: one may wonder who she/he could be in reality. However, because cheap-talk equilibria with general message structures converge to 
communication equilibria, investigating this setting would give an approximate idea of the effect of pre-play communication with more complex structures (several rounds of messages, private messages, ...). This possible research direction is left for further investigations.

\section{References}

[1] Aoyagi M., [2007], Efficient collusion in repeated auctions with communication, Journal of Economic Theory, 134, 61-92.

[2] Campbell C., [1998], Coordination in auctions with entry, Journal of Economic Theory, 82, 425-450.

[3] Chakraborty A., Harbaugh R., [2003], Cheap talk comparisons in multi-issue bargaining, Economics Letters, 78, 357-363.

[4] Compte O., [1998], Communication in repeated games with imperfect private monitoring, Econometrica, 66, 597-626.

[5] Farrell J., Gibbons R., [1987], Cheap talk can matter in bargaining, Journal of Economic Theory, 48, 221-237.

[6] Fudenberg D., J. Tirole, [1983], Sequential bargaining with incomplete information, Review of Economic Studies, 50, 221-247.

[7] Goncalves R., [2008], A communication equilibrium in English auctions with discrete bidding, Universidade Catholica Portuguesa Working paper 04/2008.

[8] Kandori M., Matsushima H., [1998], Private observation, communication and collusion, Econometrica, 66, 627-652.

[9] Matthews S., Postlewaite A., [1987], Pre-play communication in two-person sealed-bid double auctions, Journal of Economic Theory, 48, 238-263.

[10] Menezes F., Monteiro P., [2002], Auctions with endogenous participation, Review of Economic Design, 5, 71-89.

[11] Miralles A., [2010], Self-enforced collusion through comparative cheap talk in simultaneous auctions with entry, Economic Theory, 42, 523-538. 
[12] Rieck T., [2010], Signaling in first-price auctions,Bonn Econ Discussion Paper 18/2010.

[13] Samuelson W., [1985], Competitive bidding with entry costs, Economics Letters, 17, $53-57$.

[14] Sobel J., I. Takahashi, [1983], A multistage model of bargaining, Review of Economic Studies, 50, 411-426.

\section{Appendix}

Proof of Proposition 1. Let us show that $(\theta, M, \Phi)$ is a mutual best-response under conditions $(i)$ to $(i i i)$. Consider some seller $i$ whose all opponents play $(\theta, M, \Phi)$.

Bid best-response Suppose that $n$ potential bidders were selected by $\Phi$ after the message exchange, so that the maximal participating cost is $\tau_{\theta, n}$. Suppose that $i$ is active and faces $q$ active opponents. According to $\mu^{*}, i$ believes that all of them are below $\tau_{\theta, n}$.

If $q=0, i$ bids the maximal price $\rho$. If $q \geq 1$, she bids the optimal price in a firstprice procurement auction with $q$ bidders whose cost is uniformly distributed on $\left[0, \tau_{\theta, n}\right]$. By classical results, the symmetric equilibrium bid is

$$
b\left(t ; \tau_{\theta, n}, q\right):= \begin{cases}t+\frac{\tau_{\theta, n}-t}{q+1} & \text { if } q \geq 1 \\ \rho & \text { if } q=0\end{cases}
$$

If $q \geq 1$, a bidder wins the auction whenever she is the lowest bidder, namely with probability $\left(1-\frac{t}{\tau_{\theta, n}}\right)^{q}$. A bidder expected payoff is then $u\left(t ; \tau_{\theta, n}, q\right)$ with

$$
u\left(t ; \tau_{\theta, n}, q\right):= \begin{cases}\left(\frac{\tau_{\theta, n}-t}{q+1}\right)\left(1-\frac{t}{\tau_{\theta, n}}\right)^{q}-k & \text { if } q \geq 1 \\ \rho-t-k & \text { if } q=0\end{cases}
$$

Participation best-response I first establish that participation strategies are always in cutoff. The payoffs to bidder $i$ from not participating (0) and participating and loosing the auction $(-k)$ do not depend on her cost. Her payoff conditional on submitting a winning bid $b-t$ decreases with her cost. Therefore, if participating is a best response for seller $i$ at cost $t$, it still is at cost $t^{\prime}<t$. It follows that there exists some threshold $\tau_{i}$ such that $i$ participates if and only if her cost is below $\tau_{i}$. 
I now characterize the optimal cutoff $\tau_{i}$, whether $i$ is a potential bidder or not. If $i$ is a potential bidder, her optimal cutoff is $\tau_{\theta, n}$. Indeed, if $\tau_{i}>\tau_{\theta, n}, i$ will lose the auction with probability 1 for all costs in $\left.] \tau_{\theta, n}, \tau_{i}\right]$, so $\tau_{i}$ cannot be optimal. Furthermore, $\tau_{i}$ cannot be strictly smaller than $\tau_{\theta, n}$ because entry is profitable for any $t \in\left[\tau_{i}, \tau_{\theta, n}\right]$. Indeed, entry would yield to $i$ an expected payoff

$$
v\left(t, \tau_{\theta, n}, n, \frac{\tau_{\theta, n}}{\theta}\right)=(\rho-t)\left(1-\frac{\tau_{\theta, n}}{\theta}\right)^{n-1}+E\left[u\left(t, \tau_{\theta, n}, \widetilde{q}\right) \mid q \geq 1\right]-k
$$

where the first term is what $i$ would get if no opponent enters, and the second what she would get if at least one other bidder enters. Since $t<\tau_{\theta, n},(\rho-t)\left(1-\frac{\tau_{\theta, n}}{\theta}\right)^{n-1}>$ $\left(\rho-\tau_{\theta, n}\right)\left(1-\frac{\tau_{\theta, n}}{\theta}\right)^{n-1}$ which is equal to $k$ by definition of $\tau_{\theta, n}$, and $v\left(t, \tau_{\theta, n}, n, \frac{\tau_{\theta, n}}{\theta}\right)>0$. Consequently, $\tau_{i}=\tau_{\theta, n}$.

Consider now the case when $i$ is a potential deviator. Let us compute $i$ 's expected payoff in case of entry whether there is 1 or two potential bidders.

Case 1 If there is 1 potential bidder, the maximal participating cost for this potential bidder is $\rho-k$. $i$ 's expected payoff in case of entry is

$$
v\left(t, \rho-k, 2, \frac{\rho-k}{\theta}\right)=(\rho-t)\left(1-\frac{\rho-k}{\theta}\right)+\frac{(\rho-k-t)^{2}}{2 \theta}-k
$$

No potential deviator can have a higher profit than the most competitive one, who gets

$$
v\left(0, \rho-k, 2, \frac{\rho-k}{\theta}\right)=\frac{\rho-k}{\theta}\left(\theta-\frac{\rho+k}{2}\right)
$$

This payoff is negative if and only if $\theta \leq \frac{\rho+k}{2}$.

Case 2 Suppose now that two potential bidders have been selected to participate. Their maximal participating cost is $\tau(\theta, 2)$. $i$ 's expected payoff in case of entry is $v\left(t ; \tau_{\theta, 2}, 3, \frac{\tau_{\theta, 2}}{\theta}\right)$. Again, no potential deviator can have a higher profit than the most competitive one, who gets

$$
v\left(0 ; \tau_{\theta, 2}, 3, \frac{\tau_{\theta, 2}}{\theta}\right)=\rho\left(1-\frac{\tau_{\theta, 2}}{\theta}\right)^{2}+2 \frac{\tau_{\theta, 2}}{\theta}\left(1-\frac{\tau_{\theta, 2}}{\theta}\right) \frac{\tau_{\theta, 2}}{2}+\left(\frac{\tau_{\theta, 2}}{\theta}\right)^{2} \frac{\tau_{\theta, 2}}{3}-k
$$

Lemma 1. $v\left(0 ; \tau_{\theta, 2}, 3, \frac{\tau_{\theta, 2}}{\theta}\right)$ is increasing on $[0,1]$.

Proof. Replacing $\rho\left(1-\frac{\tau_{\theta, 2}}{\theta}\right)^{2}$ by $\left(\rho-\tau_{\theta, 2}\right)\left(1-\frac{\tau_{\theta, 2}}{\theta}\right)^{2}+\tau_{\theta, 2}\left(1-\frac{\tau_{\theta, 2}}{\theta}\right)^{2}$ and incorporating the equilibrium entry condition gives 


$$
v\left(0 ; \tau_{\theta, 2}, 3, \frac{\tau_{\theta, 2}}{\theta}\right)=-k \frac{\tau_{\theta, 2}}{\theta}+\frac{\tau_{\theta, 2}}{3 \theta^{2}}-\frac{\tau_{\theta, 2}^{2}}{\theta}+\tau_{\theta, 2}
$$

With $y$ standing for $\frac{\tau}{\theta}$ (and thus $\left.\frac{\partial y}{\partial \theta}=-\frac{y(1-y)}{\rho+\theta-2 \theta y}\right)$, differentiating $v\left(0 ; \tau_{\theta, 2}, 3, \frac{\tau_{\theta, 2}}{\theta}\right.$ ) with respect to $\theta$ gives

$$
\frac{\partial v\left(0 ; \tau_{\theta, 2}, 3, \frac{\tau_{\theta, 2}}{\theta}\right)}{\partial \theta}=\frac{y}{\rho+\theta-2 \theta y}\left[(1-y)\left(\rho+k-\rho \frac{y^{2}}{3}\right)+\frac{y^{2}}{3}(2-y)(\rho-\theta)\right]
$$

which is clearly positive since $y<1$.

Lemma 2. $v\left(0 ; \tau_{\theta, 2}, 3, \frac{\tau_{\theta, 2}}{\theta}\right)>0$ for $\theta=\frac{\rho+k}{2}$.

Proof. I can rewrite $v\left(0 ; \tau_{\theta, 2}, 3, \frac{\tau_{\theta, 2}}{\theta}\right)=\frac{1}{\theta^{2}}\left(\frac{\tau_{\theta, 2}^{3}}{3}+\theta \tau_{\theta, 2}\left(\theta-\tau_{\theta, 2}-k\right)\right)$. By basic calculations, if $\theta=\frac{\rho+k}{2}, \tau_{\theta, 2}=\frac{\rho-k}{2}$. Hence $v\left(0 ; \tau_{\theta, 2}, 3, \frac{\tau_{\theta, 2}}{\theta}\right)$ is positive for $\theta=\frac{\rho+k}{2}$.

Lemma 3. There exists $\underline{k}$ such that for all $k \geq \underline{k}, v\left(0 ; \tau_{\theta, 2}, 3, \frac{\tau_{\theta, 2}}{\theta}\right)<0$ for $\theta=\rho-k$.

Proof. Let us show that $v\left(0 ; \tau_{\theta, 2}, 3, \frac{\tau_{\theta, 2}}{\theta}\right)$ is negative for $\theta=\rho-k$ if and only if $\rho$ is smaller than some value $\bar{\rho} \in] 2.5 k, 3 k[$.

$v\left(0 ; \tau_{\theta, 2}, 3, \frac{\tau_{\theta, 2}}{\theta}\right)$ can be rewritten as

$$
v\left(0 ; \tau_{\theta, 2}, 3, \frac{\tau_{\theta, 2}}{\theta}\right)=\frac{\tau_{\theta, 2}}{3 \theta^{2}}\left(\tau_{\theta, 2}^{2}-3 \theta \tau_{\theta, 2}+3 \theta(\theta-k)\right)
$$

It follows that $v\left(0 ; \tau_{\theta, 2}, 3, \frac{\tau_{\theta, 2}}{\theta}\right) \leq 0$ for $\theta=\rho-k$ if and only if $f(\rho) \leq 0$, with

$$
f(\rho):=\tau(\rho)^{2}-3(\rho-k) \tau(\rho)+3(\rho-k)(\rho-2 k)
$$

and

$$
\tau(\rho):=\frac{1}{2}\left(2 \rho-k-\sqrt{k^{2}+4 k(\rho-k)}\right)
$$

Using the fact that $\tau^{\prime}(\rho)=1-\frac{k}{\sqrt{k^{2}+4 k(\rho-k)}}$ and simplifying, I get

$$
f^{\prime}(\rho)=-\tau+2 \rho-5 k-\frac{k}{\sqrt{k^{2}+4 k(\rho-k)}}(2 k-\rho)
$$

and

$$
f^{\prime \prime}(\rho)=1+\frac{2 k}{\sqrt{k^{2}+4 k(\rho-k)}}+\frac{2 k}{k+4(\rho-k)}(2 k-\rho)
$$

Clearly, $f^{\prime \prime}(\rho)$ decreases with $\rho$. For $\rho=3 k, \tau=k$ and $f^{\prime \prime}(3 k)=1+\frac{2}{3}-\frac{2}{9} k>0$. Thus $f^{\prime \prime}(\rho)$ is positive on $[k, 3 k]$. It follows that $f^{\prime}$ is strictly increasing on $[k, 3 k]$. Yet for $\rho=k, \tau=0$ 
and $f^{\prime}(k)=-4 k$, and for $\rho=3 k, \tau=k$ and $f^{\prime}(3 k)=\frac{k}{3}$. Hence there exists $\left.\widetilde{\rho} \in\right] k, 3 k[$ such that $f^{\prime}$ is negative on $[k, \widetilde{\rho}]$ and positive otherwise. The function $f$ is thus decreasing on $[k, \widetilde{\rho}]$ and increasing on $[\widetilde{\rho}, 3 k]$. I have $f(k)=0$ and $f(3 k)=k^{2}$. Therefore, there exists some value $\bar{\rho}$ in $] \widetilde{\rho}, 3 k[$ such that $f$ is negative on $[k, \bar{\rho}]$ and positive above $\bar{\rho}$. This value is defined by $f(\bar{\rho})=0$. Since $f(2.5 k)<0$, I know that $\bar{\rho} \in] 2.5 k, 3 k[$.

By Lemmas 1,2 , and 3 , there exists $\underline{k}$ defined $v\left(0 ; \tau_{\rho-k, 2}, 3, \frac{\tau_{\rho-k, 2}}{\rho-k}\right)=0$ such that if $k \geq \underline{k}$, there exists $\bar{\theta} \in\left[\rho-k, \frac{\rho+k}{2}\left[\right.\right.$ such that $v\left(0 ; \tau_{\theta, 2}, 3, \frac{\tau_{\theta, 2}}{\theta}\right) \leq 0$ for all $\theta \in[\rho-k, \bar{\theta}]$.

Message best-response If $\theta \geq \rho-k$, sellers above $\theta$ are out-bidders, who never participate and are therefore indifferent between all message strategies. This is the case also for sellers in $[\rho-k, \theta]$. Suppose now that $i$ 's cost is below $\rho-k$. Under condition $\mathbf{C} 1$, sending a message in $\mathcal{M} \backslash M$ entails a probability 0 of being selected by $\Phi$. Since potential deviators have no incentive to enter under conditions $\mathbf{C 2}$ and $\mathbf{C 3}$, the support of $i$ 's message strategy has to be M.

Finally, under condition $\mathbf{C 1}$, and provided that $i$ 's opponents follow the strategy $(\theta, M, \Phi)$, all messages in $M$ induce the same distribution function on the set of entry coalitions. It follows that $i$ is also willing to randomize over all messages in $M$.

Proof of Proposition 4. Let us express the surplus at some $(\theta, \Phi)$-equilibrium as a function of $\theta$. By definition,

$$
W^{(\theta, \Phi)}=\sum_{n=1}^{3} P^{(\theta, \Phi)}(n) w(\theta, n)
$$

The distribution function induced by $\Phi$ is

$$
\begin{aligned}
& P^{(\theta, \Phi)}(1)=\theta\left[3-3 \theta+\theta^{2}-3 \theta(1-\theta) f_{2}-\theta^{2}\left(f_{3}^{2}+f_{3}^{3}\right)\right] \\
& P^{(\theta, \Phi)}(2)=\theta^{2}\left[3(1-\theta) f_{2}^{2}+\theta f_{3}^{2}\right] \\
& P^{(\theta, \Phi)}(3)=\theta^{3} f_{3}^{3}
\end{aligned}
$$

With $z(\theta, n):=\theta^{n} w(, \theta, n),(2)$ rewrites

$$
W^{(\theta, \Phi)}=\left[3-3 \theta+\theta^{2}-3 \theta(1-\theta) f_{2}-\theta^{2}\left(f_{3}^{2}+f_{3}^{3}\right)\right] z(\theta, 1)+\left[3(1-\theta) f_{2}^{2}+\theta f_{3}^{2}\right] z(\theta, 2)+f_{3}^{3} z(\theta, 3)
$$


The aim is to show that $W^{(\theta, \Phi)}$ is decreasing on $[\rho-k, \bar{\theta}]$. Let us compute $\partial W^{(\theta, \Phi)} / \partial \theta$. Since $\tau_{\theta, 1}=\rho-k, w(\theta, 1)=\frac{(\rho-k)^{2}}{2 \theta}$ and $z(\theta, 1)$ does not depend on $\theta$. Differentiating with respect to $\theta$ gives then

$\frac{\partial W^{(\theta, \Phi)}}{\partial \theta}=\left[-3+2 \theta-3 f_{2}^{2}(1-2 \theta)-2 \theta\left(f_{3}^{2}+f_{3}^{3}\right)\right] z(\theta, 1)+\left[-3 f_{2}^{2}+f_{3}^{2}\right] z(\theta, 2)+\left[3(1-\theta) f_{2}^{2}+\theta f_{3}^{2}\right] z^{\prime}(\theta, 2)+f_{3}^{3} z^{\prime}(\theta, 3)$

Remark that $\left\{\begin{array}{l}z^{\prime}(\theta, n)=n g\left(n, \tau_{\theta, n}\right) \\ z(\theta, n-1)=g\left(n, \tau_{\theta, n-1}\right)\end{array}\right.$ with $g(n, x)=\theta^{n-2}(n-1)\left[\int_{0}^{x}(\rho-t)\left(1-\frac{t}{\theta}\right)^{n-2} d t-k x\right]$

This allows to rewrite

$$
\frac{\partial W^{(\theta, \Phi)}}{\partial \theta}=A g\left(2, \tau_{\theta, 1}\right)+B g\left(3, \tau_{\theta, 2}\right)+C g\left(2, \tau_{\theta, 2}\right)+D g\left(3, \tau_{\theta, 3}\right)
$$

with

$$
\begin{aligned}
& A:=-3+2 \theta-3 f_{2}^{2}(1-2 \theta)-2 \theta\left(f_{3}^{2}+f_{3}^{3}\right) \\
& B:=-3 f_{2}^{2}+f_{3}^{2} \\
& C:=6(1-\theta) f_{2}^{2}+2 \theta f_{3}^{2} \\
& D:=3 f_{3}^{3}
\end{aligned}
$$

To simplify this expression, remark first that $g\left(3, \tau_{\theta, 3}\right)<g\left(3, \tau_{\theta, 2}\right)$. Indeed, $g\left(3, \tau_{\theta, 3}\right)-g\left(3, \tau_{\theta, 2}\right)$ signs like $\int_{\tau_{\theta, 3}}^{\tau_{\theta, 2}}\left[k-(\rho-t)\left(1-\frac{t}{\theta}\right)\right] d t$. Yet $(\rho-t)\left(1-\frac{t}{\theta}\right)>\left(\rho-\tau_{\theta, 2}\right)\left(1-\frac{\tau_{\theta, 2}}{\theta}\right)=k$ for all for $t<\tau_{\theta, 2}$. Hence the result.

Second, using the fact that $k=\left(\rho-\tau_{\theta, 2}\right)\left(1-\frac{\tau_{\theta, 2}}{\theta}\right)$, one shows easily that

$$
\begin{aligned}
& g\left(2, \tau_{\theta, 1}\right)=\frac{\tau_{\theta, 2}^{2}}{2}(1+x)^{2} \\
& g\left(2, \tau_{\theta, 2}\right)=\tau_{\theta, 2}^{2}\left(\frac{1}{2}+x\right) \\
& g\left(3, \theta_{\theta, 2}\right)=\tau_{\theta, 2}^{2}\left(\theta-\frac{\rho}{3}-\frac{4}{3} \theta x\right)
\end{aligned}
$$

with $x:=\frac{\rho-\tau_{\theta, 2}}{\theta}$. In the following, I will use the fact that $x$ is larger than 1 whenever $\theta$ is smaller than $\frac{\rho+k}{2}$. It follows that

$$
\frac{\partial W^{(\theta, \Phi)}}{\partial \theta}<\tau_{\theta, 2}^{2} \Delta(\theta):=\tau_{\theta, 2}^{2}\left(-\left(\frac{3}{2}-\theta\right)(1+x)^{2}+3 f_{2}^{2} h_{0}+f_{3}^{2} h_{1}+f_{3}^{3} h_{2}\right)
$$

with

$$
\begin{aligned}
& h_{0}=x^{2}\left(\theta-\frac{1}{2}\right)+x\left(1-\frac{4}{3} \theta\right)+\frac{\rho}{3}-\theta+\frac{1}{2} \\
& h_{1}=-\theta x^{2}+x \theta \frac{4}{3}+\theta-\frac{\rho}{3}
\end{aligned}
$$




$$
h_{2}=-\theta x^{2}+2 \theta x+2 \theta-\rho
$$

Remark that $\left\{\begin{array}{l}-\left(\frac{3}{2}-\theta\right)(1+x)^{2}+h_{2}=-\frac{3}{2}(x-1)(x+2 \theta-1)+(\theta x-\rho) \leq 0 \forall x>1 \\ -\left(\frac{3}{2}-\theta\right)(1+x)^{2}+3 h_{0}+h_{2}=-3(1-\theta) x^{2} \leq 0\end{array}\right.$

Let us show that $\Delta(\theta)<0$ for all $\theta \leq \bar{\theta}$. The fact that $h_{1}<h_{2}$ implies that

$$
\Delta(\theta)<-\left(\frac{3}{2}-\theta\right)(1+x)^{2}+3 f_{2}^{2} h_{0}+\left(f_{3}^{2}+f_{3}^{3}\right) h_{2}
$$

- When $h_{0}<0, \Delta(\theta)$ is smaller than $-\left(\frac{3}{2}-\theta\right)(1+x)^{2}+\max \left\{0, h_{2}\right\}$, which is negative.

- Consider now the case $h_{0}>0$.

For selection functions such that $f_{3}^{1} \leq f_{2}^{1}, f_{2}^{2} h_{0}<\left(f_{3}^{2}+f_{3}^{3}\right) h_{0}$ and $\Delta(\theta)$ is smaller than $-\left(\frac{3}{2}-\theta\right)(1+x)^{2}+f_{2}^{2}\left(3 h_{0}+h_{2}\right)$ which is negative.

Consider now selection functions such that $f_{3}^{1}>f_{2}^{1}$.

If $h_{2}>0$, then $\Delta(\theta)<-\left(\frac{3}{2}-\theta\right)(1+x)^{2}+3 h_{0}+h_{2}$, and is therefore negative by the previous argument.

If $h_{2}<0$, then $\Delta(\theta)<-\left(\frac{3}{2}-\theta\right)(1+x)^{2}+3 h_{0}:=X(\theta)$.

The rest of the proof is basic calculations to show that $X(\theta)=x^{2}(4 \theta-3)-2 \theta x+\rho-2 \theta$ increases with $\theta$ and $X(\bar{\theta})<0$.

$$
X^{\prime}(\theta)=2 x^{\prime}(4 \theta x-3 x-\theta)+4 x^{2}-2 x-2
$$

Calculations show that $x^{\prime}=-\frac{x(1+x)}{\theta-\tau+\theta x}$ and that $x^{\prime}$ is negative. Therefore,

- If $4 \theta x-3 x-\theta<0, X^{\prime}(\theta)>4 x^{2}-2 x-2$ which is clearly positive for $x>1$.

- If $4 \theta x-3 x-\theta>0$, the fact that $x^{\prime}>-\frac{1+x}{\theta}$ implies that

$$
X^{\prime}(\theta)>-2 \frac{1+x}{\theta}(4 \theta x-3 x-\theta)+4 x^{2}-2 x-2=\frac{2 x}{\theta}((2 x+3)(1-\theta)+x-\theta)>0
$$

Finally, for $\theta=\frac{\rho+k}{2}, x=1$, so that $X\left(\frac{\rho+k}{2}\right)=\rho-3$. This is negative whenever $\rho \leq 3$. Therefore, $\rho \leq 3$ is a sufficient condition for $W^{(\theta, \Phi)}$ to decreases with $\theta \leq \bar{\theta}$. Note that the upper bound 3 for $\rho$ is not tight. 